\title{
Influencing GDP's intentions to remove third molars
}

\author{
Can psychological models bridge the gap between clinical guidelines and clinicians' behaviour? A randomised controlled \\ trial of an intervention to influence dentists' intention to implement evidence-based practice. D. Bonetti, M. Johnston,
} N. B. Pitts, C. Deery, I. Ricketts, M. Bahrami, C. Ramsay and J. Johnston Br Dent J 2003; 195: 602-606

\section{Objective}

The lag between publication of evidence for clinical practice and implementation by clinicians may be decades. Research using psychological models demonstrates that changing intention is very important in changing behaviour. This study examined an intervention (rehearsing alternative actions) to change dentists' intention to implement evidence-based practice (EBP) for third molar (TM) management.

Design

Randomised controlled trial / postal in primary care.

Subjects and methods

Dentists were randomly selected from the Scottish Dental Practice Board Register, then randomly allocated to intervention or control groups, and sent a questionnaire. The intervention group listed management alternatives to TM extraction prior to their TM extraction intention, and the control group did not. Based on psychological models for reducing a behaviour's frequency (EBP is weighted against TM extraction), prior listing of alternatives should decrease extraction intention.

Main outcome measure

Intention to extract TMs.

Results

A total of 99 dentists -70 Males, 29 Females; mean age $=41.42$ years $(\mathrm{SD}=8.62)$ participated in the study. The intervention significantly influenced intention to extract TMs, as desired. Despite similar background and knowledge of management alternatives, participants in the intervention group had significantly lower intention to extract: control group mean $(\mathrm{SD})=0.39$ (1.99); intervention group mean $(\mathrm{SD})=-0.78$ (1.89); mean difference $(\mathrm{SE})=1.17$ (0.42); 95\% confidence interval for the difference $=0.34$ to 1.99 .

Conclusion

Results suggest this intervention, which successfully influenced a proximal predictor of behaviour pertinent to dental EBP, may result in improved EBP in a service-level trial. Basing implementation interventions and trial methodology on psychological models may effectively bridge the gap between clinical guidelines and practice.

\section{IN BRIEF}

- Systematic reviews of guideline implementation trials have shown that increasing knowledge and skills through audit or education is generally insufficient to achieve significant changes in clinical practice.

- Since guidelines often require clinicians to change their behaviour, basing the design of implementation interventions on psychological models of behaviour change may be an effective means of improving clinical practice and patient outcomes.

- The likelihood of a successful resource-intensive service level trial may also be increased by testing the influence of the intervention on an outcome representative of evidence-based practice, derived from psychological models, in a modelling experiment.

\section{COMMENT}

Dentists, in common with other health professionals, do not always base their clinical practice on evidence, despite the benefits that this would have for quality and equity of healthcare. It is well known that publishing evidence based guidelines, even when accompanied by specific recommendations, does not automatically translate into practice. Increasing knowledge and skills are not, on their own, sufficient to change behaviour.

Over 240 controlled studies of interventions aimed at increasing the implementation of evidence based practice have had only limited success and have not produced a clear picture of effective intervention techniques. This is the background to this multidisciplinary study that has developed and evaluated a dental intervention based on the scientific study of human behaviour. Changing behaviour is a psychological issue and these researchers have brought psychological theory and techniques to bear on a question of dental practice.

The particular dental practice that was targeted is the management of impacted and un-erupted third molars, for which recent evidence based guidelines have recommended less frequent extraction. It is not easy to get people to do less of something they have been routinely doing: certainly, statements and exhortations to do a habitual behaviour less frequently have little impact. However, psychological research in other areas of clinical practice has shown that encouraging people to plan their behaviour on the basis of generating alternative future behaviours is more effective. Bonetti et al. have developed an elegantly simple intervention of asking dentists to develop alternative treatments to extraction.

The results are encouraging. Dentists in the intervention group generated an average of three treatment options. As predicted, those in the intervention group were more likely to intend to follow the third molar extraction guidelines than those in the control group.

This study uses psychological theory in a second, useful way. In preparation for developing a large-scale, service level intervention, this research group has modelled a behavioural intervention. The outcome is not behaviour, but a predictor of behaviour, that is, intended behaviour. Only if effectiveness of the intervention can be demonstrated in this way is it worthwhile to continue with a more ambitious and expensive trial. Increasingly, such an approach is regarded as good practice, especially by funding bodies such as the Medical Research Council.

This timely study makes an important contribution to developing methods to increase evidence based practice. Future replication studies are needed, using methods that increase study participation rates and applying the technique to other behaviours. If the reliability and generalizability of these results are established, the application of this technique to dentistry should increase the quality of dental practice.

Susan Michie, Reader in Clinical Health Psychology, University College London doi:10.1038/sj.bdj.4810563 